\title{
The Effect of Early Rescue ICSI and Split IVF-ICSI in Preventing Low Fertilization Rate During the First ART Cycle: A Real-world Retrospective Cohort Study
}

Dongzi Yang ( $\sim$ yangfuchanke@163.com )

Sun Yat-sen Memorial Hospital of Sun Yat-sen University https://orcid.org/0000-0001-9890-219X

Linlin Jiang

Sun Yat-Sen Memorial Hospital

Yifan Qian

Sun Yat-Sen Memorial Hospital

Xiaoli Chen

Sun Yat-Sen Memorial Hospital

Xiaohui Ji

Sun Yat-Sen Memorial Hospital

\section{Songbang Ou}

Sun Yat-Sen Memorial Hospital

Ruiqi Li

Sun Yat-Sen Memorial Hospital

Yu Li

Sun Yat-Sen Memorial Hospital

\section{Research}

Keywords: short gamete coincubation, early rescue ICSI, split IVF-ICSI, low fertilization, first ART cycle

Posted Date: July 28th, 2021

DOI: https://doi.org/10.21203/rs.3.rs-714068/v1

License: (c) (i) This work is licensed under a Creative Commons Attribution 4.0 International License. Read Full License 


\section{Abstract}

Background

Short gamete coincubation in in vitro fertilization (IVF-S) combined with early rescue intracytoplasmic sperm injection (RICSI) and split IVF-ICSI insemination, are two methods to prevent unpredicted low or failed fertilization. This study aimed to determine the utility of IVF-S combined with R-ICSI and split IVF-ICSI during the first assisted reproductive technology (ART) cycle.

Patients and methods

A single-center retrospective cohort study based on real-world data. Couples with a high risk of low IVF fertilization during the first ART cycle underwent IVF-S with R-ICSI $(n=191)$ or split IVF-ICSI $(n=775)$. Fertilization rate, embryo quality, and clinical outcomes were measured.

Results

After propensity score matching, we included 188 couples in the IVF-S with R-ICSI group as Group 1 and 720 in the split IVF-ICSI group as Group 2, with low IVF fertilization rates of $4.79 \%$ and $9.03 \%$, respectively. Normal fertilization rates were similar in the two groups; however, Group1 had a higher multiple pronuclei rate $(10.42 \%$ vs. $4.50 \%, P<0.001)$. The groups were similar for rates of high-quality embryos, blastocyst formation rate, embryo implantation, clinical pregnancy, miscarriage, loss to follow-up, and live birth. Low fertilization following IVF occurred in nine couples in Group 1 with 47 MII oocytes performed R-ICSI and 65 in Group 2 with 331 MII oocytes performed ICSI. Similar fertilization rate, embryo development, and clinical outcomes were noted in two groups with low fertilization.

Conclusions

IVF-S with early R-ICSI and split IVF-ICSI were effective strategies in preventing IVF fertilization failure or low fertilization rate in couples with high-risk factors. IVF-S with early R-ICSI could become the preferred approach because of its advantages-fewer ICSI procedures, similar clinical pregnancy rate and live birth rate.

\section{Background}

The incidence of total fertilization failure in conventional in vitro fertilization (IVF) is about 3.52-20.00\% [1][2][3], often leading to cycle cancellation. However, currently available diagnostic strategies are limited. Late rescue intracytoplasmic sperm injection (ICSI) at 16-18 h after fertilization could achieve better fertilization and cleavage rates, but subsequent embryonic development and pregnancy rates are not ideal due to eggs aging [4]. There are presently two methods to prevent unpredicted low or failed fertilization, short gamete coincubation in IVF (IVF-S) combined with early rescue ICSI (R-ICSI) and IVF-ICSI split insemination.

Oocytes have been observed to be fertilized $2 \pm 4 \mathrm{~h}$ after exposure to spermatozoa [5][6], and the second polar body is released in $\sim 90 \%$ of fertilized oocytes by $6 \mathrm{~h}$ using time-lapse video cinematography [7]. Short gamete coincubation while observing for early signs of fertilization makes it possible to perform early R-ICSI attempts before the time-related deterioration in oocyte quality [8][9][10][11]. However, to facilitate this observation, the granulosa cells around the oocyte should be removed under the microscope. Studies showed that IVF-S had diverse effects on fertilization and embryo quality. Some studies showed that IVF-S could reduce the adverse effects of the semen and its metabolites on the embryos, improving embryo quality and the clinical pregnancy rate [12][13]. Also, it has been reported that granulosa cell removal after six hours of short gamete coincubation had a lower abnormal fertilization rate than if they were removed at 20 hours of fertilization [14]. However, some studies reported that early removal of granulosa cells increased the 
multiple-fertilization rate [15][16][17]. Even so, most researchers believe that granulosa cell removal at six hours of short gamete coincubation enables early discovery of low or failed fertilization with no effect on embryo quality [14][17][18].

IVF-ICSI split insemination is another method to prevent fertilization failure [19][20]. Failed fertilization after IVF occurred in ten of 60 couples (16.7\%) with unexplained infertility, but in none, if ICSI was performed with conventional IVF on sibling oocyte cumulus complexes (COCs) [19]. A committee opinion indicated that ICSI for unexplained infertility, without male factor infertility, was associated with a higher fertilization rate; however, it did not improve live-birth outcomes [21]. Some studies reported that IVF-ICSI split insemination increased the fertilization rate and decreased the rates of complete fertilization failure in cases of mild male factor infertility [22] and polycystic ovary syndrome (PCOS) patients [23]. Although fertilization rate is higher through split IVF-ICSI, the blastocyst formation rate obtained following ICSI was lower than after IVF [24].

In the absence of clinical guidelines on the insemination method in couples with a high risk of fertilization failure in conventional IVF, we aimed to assess the effectivity of IVF-S combined with early R-ICSI and split IVF-ICSI, trying to succeed in at least some cases of unpredictable fertilization failure, by real-world retrospective cohort study [25].

\section{Patients And Methods}

\section{Participants}

A single-center retrospective cohort study was performed, analyzing data of patients who underwent IVF-S with early RICSI (Group 1) or split IVF-ICSI (Group 2) at the Sun Yat-sen Memorial Hospital from January 2017 to July 2019. All patients were during their first assisted reproductive technologies (ART) cycle with four or more retrieved oocytes and at least one of the high-risk factors for poor fertilization: infertility for more than five years, borderline semen parameters, or unexplained infertility. The diagnosis of unexplained infertility was based on the following criteria: regular ovulatory cycles, normal uterine cavity and patent fallopian tubes, no clinical or sonographic evidence of endometriosis, and normal semen parameters following the World Health Organization criteria. Patients with oocyte maturation disorder were excluded. Sun Yat-sen Memorial Hospital ethical review board approved the study. The data were anonymized, and the requirement for informed consent was therefore waived.

\section{Clinical Procedures}

All participants were treated with a gonadotropin-releasing hormone $(\mathrm{GnRH})$ agonist or $\mathrm{GnRH}$ antagonist protocol. Individually adapted doses of the recombinant or highly purified follicle-stimulating hormone were administered according to age and ovarian reserve, followed by adjusted dosage based on serum estradiol levels and follicular development, as indicated by ultrasonographic monitoring. Human chorionic gonadotropin (hCG) or GnRH agonist were administered to induce oocyte maturation when at least three follicles reached a mean diameter of $16 \mathrm{~mm}$, or at least two follicles were $18 \mathrm{~mm}$ in diameter or larger. Oocytes were retrieved $36 \mathrm{~h}$ after triggering.

Semen was collected into sterile containers by masturbation and then kept for 30 minutes at $37^{\circ} \mathrm{C}$. After liquefaction, samples were analyzed for sperm concentration and motility following the World Health Organization criteria. After liquefaction, sperms were selected by gradient centrifugation (Vitrolife, Sweden AB) and centrifuged at 500×g for 15 minutes. The sperm pellet was collected and washed in SpermRinse (Vitrolife, Sweden AB) at 300×g for 5 minutes and then IVF medium at $300 \times \mathrm{g}$ for 5 minutes. The final sperm pellet was resuspended in $1 \mathrm{~mL}$ of IVF medium for insemination.

Before co-incubation of the gametes, the rate of the progressive sperm cells was evaluated and the oocytes were inseminated with $1.0 \times 10^{5} / \mathrm{ml}$ progressive sperm cells $3-5 \mathrm{~h}$ after oocyte retrieval. In Group 1 , four to five 
oocytes/zygotes, considered mature oocytes, with relatively loose cumulus cell layers, were selected by an experienced embryologist and transferred into GMOPS medium (Vitrolife, Sweden AB) for removing the cumulus cells $5 \mathrm{~h}$ postinsemination. Then the oocytes were transferred into G-IVF medium (Vitrolife, Sweden AB) for polar body observation by inverted microscope (Nikon, Japan). The presence of two polar bodies indicated barrier-free fertilization. If two or more oocytes were fertilized, the remaining oocytes/zygotes were cultured as in conventional IVF. If less than two metaphase II (MII) oocytes presented two polar bodies, second evaluation for two polar body was done one more hour later. This study defined fertilization rate $<30 \%$, including total fertilization failure, as a low fertilization rate [26][27]. When a low fertilization rate was identified, early R-ICSI was performed $6 \mathrm{~h}$ post-insemination, which procedure was as same as regular ICSI, using the rest of the semen. COCs were randomly selected for ICSI or IVF in Group 2. The COCs were preincubated for 3-6 $\mathrm{h}$ before insemination or injection. Conventional IVF and ICSI were performed according to the laboratory's routine insemination procedures. The oocytes in the standard incubation time group were co-incubated with sperm in $1 \mathrm{~mL}$ of IVF medium for $16-18 \mathrm{~h}$.

Embryo quality was classified based on the embryo morphology following the Istanbul consensus workshop on embryo assessment [28]. D3 cleavage embryos or blastocysts were transferred. All patients received the luteal support with progesterone from the day of oocyte retrieval. On Day $14 \pm 3$ after ET, participants underwent a pregnancy test (serum measurement of $\boldsymbol{\beta}$-hCG). Clinical pregnancy was confirmed through ultrasonographic observation of the intrauterine gestation sac, the fetal pole, and cardiac activity at 6-7 weeks of gestation. If pregnancy was confirmed, luteal phase support was continued for up to ten weeks of gestation. The neonatal outcome data were obtained by telephone interview with the parents after delivery.

\section{Statistical analysis}

Case matching: The groups differed in age and sperm concentration after recruiting based on the inclusion and exclusion criteria. We included the female age and sperm concentration in 1:4 propensity score matching (PSM) to eliminate the influence of baseline differences in this real-world study, as these might act as confounding factors.

Statistical method: R 3.5.3 software (The University of Auckland, Auckland city, New Zealand) was used for PSM. IBM SPSS Statistics for Windows, Version 24.0 software (IBM Corp., Armonk, NY, USA) was used for data analysis. PASS(Power Analysis and Sample Size) 15.0.5 was used for power analysis based on sample size and live birth rate of two group. Continuous variables are expressed as mean \pm standard deviation, and the independent-sample $t$-test compared the groups. Count data are expressed as percentage (\%), and the groups were compared for rates by the $\chi 2$ test or Fisher's exact test. Differences with $P<0.05$ were considered statistically significant.

\section{Results}

We identified 191 cases of Group 1 and 775 cases of Group 2 based on the inclusion and exclusion criteria. After PSM, Group 1 comprised 188 cases, and group 2 consisted of 720 cases, as shown in Table 1. The data in Table 1 show that Group 2 had a longer infertility period, but the proportion of primary infertility was lower than in Group 1 . The proportion of $\mathrm{GnRH}$ agonist protocol was higher in Group 1 compared with Group 2. The mean patient age, etiology of infertility, baseline serum FSH, AMH and sperm concentration and motility on oocyte retrieval day were similar between the groups after PSM. 
Table 1

Demographic characteristics of the patients in the two study groups

\begin{tabular}{|c|c|c|c|c|c|c|}
\hline & \multicolumn{2}{|l|}{ Before PSM } & \multirow{3}{*}{$\begin{array}{l}P \\
\text { value }\end{array}$} & \multicolumn{2}{|l|}{ After PSM } & \multirow{3}{*}{$\begin{array}{l}P \\
\text { value }\end{array}$} \\
\hline & Group 1 & Group 2 & & Group1 & Group 2 & \\
\hline & $N=191$ & $N=775$ & & $N=188$ & $N=720$ & \\
\hline Age (y) & $31.37 \pm 3.85$ & $32.34 \pm 3.96$ & 0.002 & $31.46 \pm 3.79$ & $31.94 \pm 3.72$ & 0.112 \\
\hline Years of infertility (y) & $4.89 \pm 2.66$ & $5.96 \pm 3.20$ & 0.000 & $4.93 \pm 2.66$ & $5.89 \pm 3.15$ & 0.000 \\
\hline Primary infertility (\%) & 78.53(150/191) & 67.48(523/775) & 0.003 & 78.72(148/188) & 69.58(501/720) & 0.013 \\
\hline \multicolumn{7}{|l|}{$\begin{array}{l}\text { Infertility factors, \% } \\
(n)\end{array}$} \\
\hline $\begin{array}{l}\text { Ovulation } \\
\text { dysfunction }\end{array}$ & $9.42(18 / 191)$ & $10.71(83 / 775)$ & \multirow[t]{7}{*}{0.131} & $9.57(18 / 188)$ & 10.98(79/720) & \multirow[t]{7}{*}{0.089} \\
\hline Tubal factor & 21.99(42/191) & 26.58(206/775) & & $21.28(40 / 188)$ & 27.5(198/720) & \\
\hline Endometriosis & $4.71(9 / 191)$ & $3.23(25 / 775)$ & & $4.79(9 / 188)$ & $3.06(22 / 720)$ & \\
\hline Mild male factor & 16.75(32/191) & 15.23(118/775) & & 16.49(31/188) & $14.58(105 / 720)$ & \\
\hline Unexplained factor & 27.74(53/191) & 22.19(172/775) & & $28.19(53 / 188)$ & $22.50(162 / 720)$ & \\
\hline $\begin{array}{l}\text { Multiple female } \\
\text { factor }\end{array}$ & $10.99(21 / 191)$ & $8.13(63 / 775)$ & & $11.17(21 / 188)$ & $7.92(57 / 720)$ & \\
\hline $\begin{array}{l}\text { Both female and } \\
\text { male }\end{array}$ & $8.38(16 / 191)$ & 13.94(108/775) & & $8.51(16 / 188)$ & $13.47(97 / 720)$ & \\
\hline Basal FSH (U/L) & $8.05 \pm 3.33$ & $7.88 \pm 3.91$ & 0.164 & $8.06 \pm 3.35$ & $7.83 \pm 3.90$ & 0.458 \\
\hline AMH (ng/ml) & $5.03 \pm 3.85$ & $5.40 \pm 3.83$ & 0.372 & $4.98 \pm 3.79$ & $5.54 \pm 3.87$ & 0.151 \\
\hline \multicolumn{7}{|l|}{ COS protocol, \% (n) } \\
\hline GnRH agonist & 73.82(141/191) & $65.16(505 / 775)$ & \multirow[t]{2}{*}{0.023} & $73.94(139 / 188)$ & $64.86(467 / 720)$ & \multirow[t]{2}{*}{0.019} \\
\hline GnRH antagonist & $26.18(50 / 191)$ & $34.84(270 / 775)$ & & 26.06(49/188) & $35.14(253 / 720)$ & \\
\hline $\begin{array}{l}\text { Duration of } \\
\text { stimulation }\end{array}$ & $11.38 \pm 2.47$ & $11.01 \pm 2.87$ & 0.102 & $11.40 \pm 2.48$ & $11.01 \pm 2.93$ & 0.097 \\
\hline Gn (IU) & $2109 \pm 865$ & $2003 \pm 774$ & 0.097 & $2122 \pm 864$ & $1982 \pm 768$ & 0.03 \\
\hline $\begin{array}{l}\text { Semen } \\
\text { concentration }(\mathrm{M} / \mathrm{mL})\end{array}$ & $63.07 \pm 36.39$ & $56.63 \pm 35.04$ & 0.02 & $61.73 \pm 33.97$ & $57.74 \pm 33.34$ & 0.145 \\
\hline Semen motility (\%) & $55.47 \pm 12.15$ & $53.87 \pm 12.80$ & 0.118 & $55.61 \pm 11.74$ & $53.88 \pm 12.84$ & 0.093 \\
\hline $\begin{array}{l}\text { Sperm total motile } \\
\text { count }(M)\end{array}$ & $94.48 \pm 74.07$ & $85.75 \pm 81.40$ & 0.177 & $93.78 \pm 73.35$ & $87.44 \pm 81.23$ & 0.332 \\
\hline $\begin{array}{l}\text { Data are expressed as } \\
0.05) \text {. PSM, propensity } \\
\text { hormone; COS, control } \\
\text { vitro fertilization (IVF) }\end{array}$ & $\begin{array}{l}\text { on } \pm \text { standard } \\
\text { ore matching; } 1 \\
\text { ovarian hypers } \\
\text { h early rescue i }\end{array}$ & $\begin{array}{l}\text { ation or percente } \\
\text { illion; basal FS } \\
\text { ulation; Gn, Gone } \\
\text { cytoplasmic spe }\end{array}$ & $\begin{array}{l}\text { Bold } \\
\text { asal f } \\
\text { tropin } \\
\text { inject }\end{array}$ & $\begin{array}{l}\text { ts highlight sta } \\
\text { le stimulating I } \\
\text { oup 1, short ga } \\
\text { (ICSI); Group 2, }\end{array}$ & $\begin{array}{l}\text { cal significance } \\
\text { oe; AMH, Anti-m } \\
\text { e coincubation d } \\
\text { t IVF and ICSI. }\end{array}$ & $\begin{array}{l}< \\
\text { llerian } \\
\text { ing in }\end{array}$ \\
\hline
\end{tabular}

In Group 1, five cases were under total IVF fertilization failure and nine cases were under low IVF fertilization which were perfeormed early R-ICSI. In Group 2, 32 cases were under total IVF fertilization failure and 65 cases were under low IVF 
fertilization. As shown in Table 2, similar complete IVF fertilization failure (2.66\% vs. $4.44 \%$ ) and low IVF fertilization $(4.79 \%$ vs. $9.03 \%)$ rates were noted in Groups 1 and 2, respectively. The groups were also similar in the rates of normal fertilization following IVF, normal cleavage following IVF, overall normal fertilization, overall normal cleavage, d3 highquality embryos, blastocyst formation, high-quality blastocyst, embryo implantation, clinical pregnancy, miscarriage, loss to follow-up, and birth. However, rates of multiple pronuclei (PN) following IVF and overall multiple PN in Group 1 were significantly higher than in Group 2.

Table 2

Fertilization rate, embryo quality, and clinical outcomes in the study groups

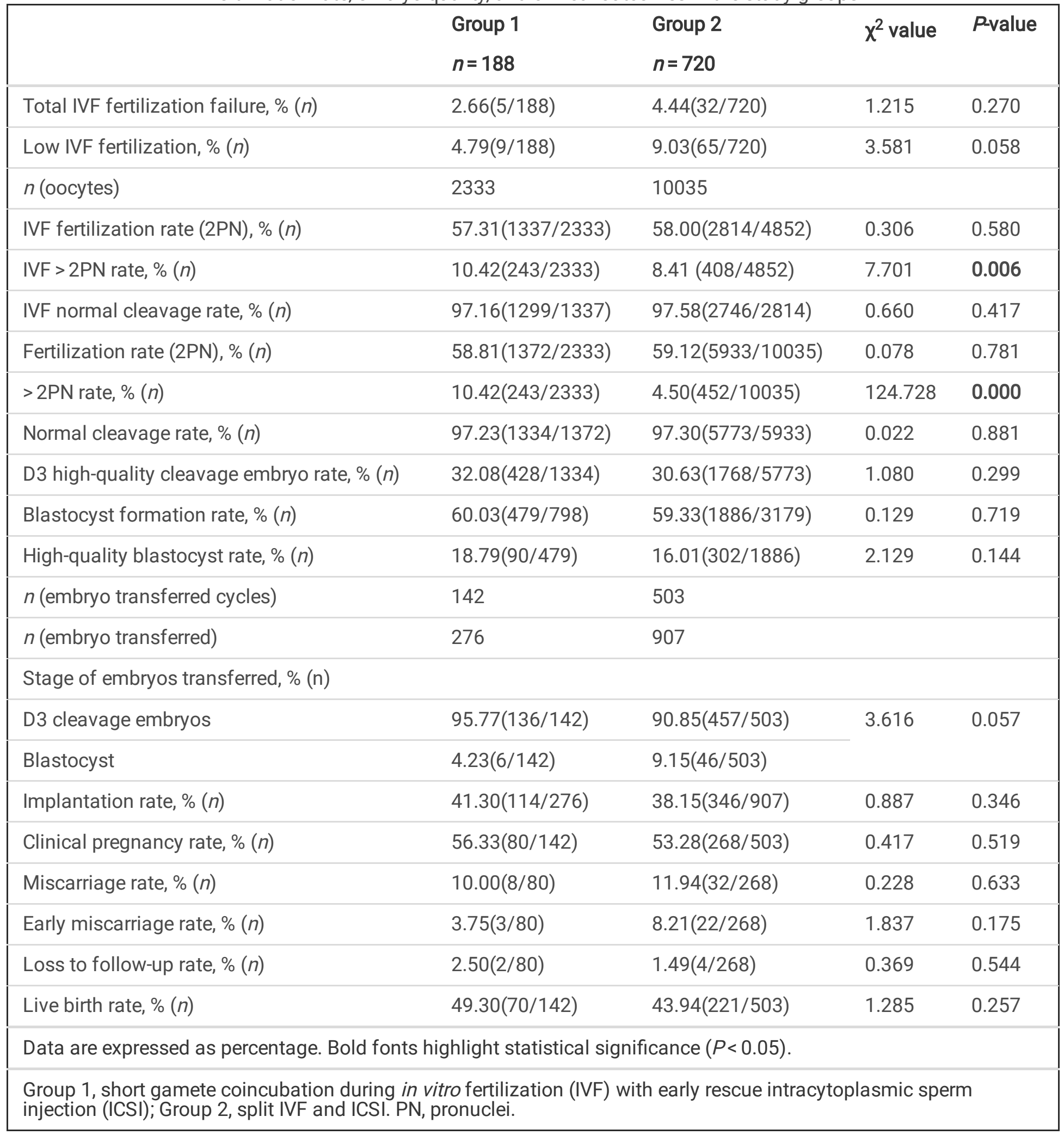


We divided the study patients into low $(n=74)$ and normal $(n=834)$ fertilization rate following IVF, using the $30 \%$ as a cut-off value. Table 3 shows the baseline characteristics of these two groups. The groups were similar for age, years of infertility, primary infertility rate, etiology of infertility, basal $\mathrm{FSH}, \mathrm{AMH}, \mathrm{COS}$ protocol, sperm concentration, and sperm motility.

Table 3

The baseline characteristics of low and normal IVF fertilization rate groups

\begin{tabular}{|c|c|c|c|}
\hline & Low IVF fertilization rate & Normal IVF fertilization rate & $P$-value \\
\hline$n$ & 74 & 834 & \\
\hline Age (y) & $31.86 \pm 3.37$ & $31.84 \pm 3.77$ & 0.959 \\
\hline Years of infertility (y) & $5.49 \pm 2.87$ & $5.71 \pm 3.09$ & 0.567 \\
\hline \multicolumn{3}{|l|}{ Infertility factors, $\%(n)$} & 0.270 \\
\hline Ovulation dysfunction & $6.76(5 / 74)$ & $11.03(92 / 834)$ & \multirow[t]{7}{*}{0.634} \\
\hline Tubal factor & $25.68(19 / 74)$ & $26.26(219 / 834)$ & \\
\hline Endometriosis & $4.05(3 / 74)$ & $3.36(28 / 834)$ & \\
\hline Mild male factor & $14.86(11 / 74)$ & $14.99(125 / 834)$ & \\
\hline Unexplained factor & $22.97(17 / 74)$ & 23.74(198/834) & \\
\hline Multiple female factor & $6.76(5 / 74)$ & $8.75(73 / 834)$ & \\
\hline Both female and male & $18.92(14 / 74)$ & $11.87(99 / 834)$ & \\
\hline Basal FSH (U/L) & $7.47 \pm 2.34$ & $7.92 \pm 3.89$ & 0.332 \\
\hline $\mathrm{AMH}(\mathrm{ng} / \mathrm{ml})$ & $6.33 \pm 4.32$ & $5.33 \pm 3.80$ & 0.079 \\
\hline \multicolumn{4}{|l|}{ CoS protocol, \% (n) } \\
\hline GnRH agonist & $59.46(44 / 74)$ & $67.39(562 / 834)$ & \multirow[t]{2}{*}{0.165} \\
\hline GnRH antagonist & $40.54(30 / 74)$ & $32.61(272 / 834)$ & \\
\hline Duration of stimulation & $10.88 \pm 2.56$ & $11.11 \pm 2.87$ & 0.499 \\
\hline Gn (IU) & $1956 \pm 811$ & $2016 \pm 789$ & 0.53 \\
\hline Sperm concentration $(\mathrm{M} / \mathrm{mL})$ & $53.61 \pm 37.80$ & $59.00 \pm 33.08$ & 0.238 \\
\hline Sperm motility (\%) & $52.50 \pm 12.96$ & $54.39 \pm 12.60$ & 0.218 \\
\hline Sperm total motile count (M) & $72.07 \pm 61.91$ & $90.24 \pm 80.92$ & 0.060 \\
\hline Data are expressed as mean & andard deviation or perc & tage. & \\
\hline
\end{tabular}

Low fertilization following IVF occurred in nine couples in Group 1 with 47 MII oocytes performed R-ICSI and 65 in Group 2 with 331 MII oocytes performed ICSI. These two subgroups were similar for basic characteristics, including age, years of infertility, primary infertility rate, causes of infertility, and semen motility, as shown in Table 4. However, sperm concentration in couples from Group 1 was slightly lower than in the couples from Group 2. The subgroups were similar 
for rates of fertilization following IVF, ICSI normal fertilization, cleavage, d3 high-quality embryos, blastocyst formation and high-quality blastocyst. Fourteen embryos were transferred in seven transfer cycles in the couples from Group 1, and 74 embryos transferred in 44 transfer cycles in the couples from Group 2. The data in Table 4 show that rates of embryo transfer cancellation, implantation, clinical pregnancy, and live birth were similar in the two subgroups. 
Table 4

Baseline characteristics, fertilization rate, embryo development, and clinical outcomes of early R-ICSI in Group 1 and ICSI in Group 2 for couples with low IVF fertilization rate

\begin{tabular}{|c|c|c|c|}
\hline & Early R-ICSI in Group 1 & ICSI in Group 2 & $P$-value \\
\hline$n$ & 9 & 65 & \\
\hline Age (y) & $32.67 \pm 4.39$ & $31.75 \pm 3.24$ & 0.451 \\
\hline Years of infertility (y) & $5.22 \pm 3.46$ & $5.53 \pm 2.81$ & 0.765 \\
\hline Primary infertility (\%) & $77.78(7 / 9)$ & $76.92(50 / 65)$ & 0.954 \\
\hline \multicolumn{4}{|l|}{ Infertility factors \%(n) } \\
\hline Ovulation dysfunction & $11.11(1 / 9)$ & $6.15(4 / 65)$ & \multirow[t]{7}{*}{1.000} \\
\hline Tubal factor & $33.33(3 / 9)$ & $24.62(16 / 65)$ & \\
\hline Endometriosis & $0(0 / 9)$ & $4.62(3 / 65)$ & \\
\hline Mild male factor & $11.11(1 / 9)$ & $15.38(10 / 65)$ & \\
\hline Unexplained factor & $22.22(2 / 9)$ & $23.08(15 / 65)$ & \\
\hline Multiple female factor & $0(0 / 9)$ & $7.69(5 / 65)$ & \\
\hline Both female and male & $22.22(2 / 9)$ & $18.46(12 / 65)$ & \\
\hline$n$ (oocytes) & 92 & 865 & \\
\hline$n$ (ICSI MII oocytes) & 47 & 331 & \\
\hline Sperm concentration $(\mathrm{M} / \mathrm{mL})$ & $30.56 \pm 21.42$ & $56.80 \pm 38.57$ & 0.007 \\
\hline Sperm motility (\%) & $47.22 \pm 14.39$ & $53.23 \pm 12.70$ & 0.194 \\
\hline Sperm total motile count (M) & $37.67 \pm 27.67$ & $76.84 \pm 63.92$ & 0.004 \\
\hline IVF fertilization rate, $\%(n)$ & $7.61(7 / 92)$ & $12.23(51 / 417)$ & 0.207 \\
\hline ICSI fertilization rate (2PN), \% (n) & $74.47(35 / 47)$ & $69.79(231 / 331)$ & 0.511 \\
\hline ICSI normal cleavage rate, $\%(n)$ & $100.00(35 / 35)$ & $97.40(225 / 231)$ & 0.335 \\
\hline ICSI fertilization rate $(>2 \mathrm{PN}), \%(n)$ & $2.13(1 / 47)$ & $0.91(3 / 331)$ & 0.444 \\
\hline D3 high-quality cleavage embryo rate, \% (n) & $38.46(15 / 39)$ & $33.59(88 / 262)$ & 0.549 \\
\hline Blastocyst formation rate, \% ( $n)$ & $20.00(2 / 10)$ & $54.37(56 / 103)$ & 0.081 \\
\hline High-quality blastocyst rate, $\%(n)$ & $0.00(0 / 2)$ & $16.07(9 / 56)$ & 1.000 \\
\hline$n$ (embryo transferred cycle) & 7 & 44 & \\
\hline$n$ (embryo transferred) & 14 & 74 & \\
\hline
\end{tabular}

Data are expressed as mean \pm standard deviation or percentage. Bold fonts highlight statistical significance $(P<$ 0.05).

ICSI, intracytoplasmic sperm injection; R-ICSI, rescue ICSI; M, million; MII, metaphase II; PN, pronuclei. Group 1, short gamete coincubation during in vitro fertilization (IVF) with early R-ICSI; Group 2, split IVF and ICSI. 


\begin{tabular}{|lllc|}
\hline & Early R-ICSI in Group 1 & ICSI in Group 2 & P-value \\
\hline D3 cleavage embryos & $100.00(7 / 7)$ & $90.91(40 / 44)$ & 1.000 \\
\hline Blastocyst & $0.00(0 / 7)$ & $9.09(4 / 44)$ & 0.540 \\
\hline Embryo transfer cancellation rate, \% $(n)$ & $22.22(2 / 9)$ & $32.31(21 / 65)$ & 0.292 \\
\hline Implantation rate, \% $(n)$ & $57.14(8 / 14)$ & $41.89(31 / 74)$ & 0.344 \\
\hline Clinical pregnancy rate, \% $(n)$ & $71.43(5 / 7)$ & $52.27(23 / 44)$ & 0.132 \\
\hline Live birth rate, \% $(n)$ & $71.43(5 / 7)$ & $40.91(18 / 44)$ & \\
\hline $\begin{array}{l}\text { Data are expressed as mean } \pm \text { standard deviation or percentage. Bold fonts highlight statistical significance }(P< \\
\text { 0.05). }\end{array}$ & & \\
\hline $\begin{array}{l}\text { ICSI, intracytoplasmic sperm injection; R-ICSI, rescue ICSI; M, million; MII, metaphase II; PN, pronuclei. Group 1, short } \\
\text { gamete coincubation during in vitro fertilization (IVF) with early R-ICSI; Group 2, split IVF and ICSI. }\end{array}$ \\
\hline
\end{tabular}

\section{Discussion}

After PSM, this study included 188 couples in IVF-S with early R-ICSI and 720 in split IVF-ICSI. Total fertilization failure occurred in 37 of 908 couples (4.07\%), and a low IVF fertilization rate was noted in 74 of 908 couples (8.15\%). Early RICSI benefited $4.79 \%$ of patients in Group 1, and ICSI benefited $9.03 \%$ of patients in Group 2. Even though Group 1 had a higher multiple pronuclei rate, the groups were similar for rates of d3 high-quality embryos, blastocyst formation, highquality blastocyst, embryo implantation, clinical pregnancy, miscarriage, loss to follow-up, and birth. Nine cases were done R-ICSI in Group 1 and 65 cases were performed spit IVF-ICSI in Group2 based on low IVF fertilization rate. Similar fertilization rate, embryo development, and clinical outcomes were noted between these two groups.

Total fertilization failure or low fertilization rate after conventional IVF is still prevalent and unpredictable. It is not surprising that we found no difference between the low and normal IVF fertilization rate groups, even though we believe that infertility for over five years, borderline sperm quality, or unexplained infertility are high-risk factors for poor fertilization. Early R-ICSI prevented complete fertilization failure. The rates of fertilization, implantation, and pregnancy in early R-ICSI after IVF-S were much higher than the so-called ICSI rescue procedure in which ICSI is performed on the second day [10]. Split IVF-ICSI randomly allocates the obtained oocytes to IVF or ICSI, preventing in part the risk of complete fertilization failure or low fertilization rate. Here, we demonstrated that early R-ICSI and split IVF-ICSI were effective strategies in preventing IVF fertilization failure or low fertilization rate in couples with high-risk factors.

Our results documented that normal fertilization rates in the two groups were similar; however, a higher multiple PN rate was noted in Group 1 (10.42\% vs. $8.41 \%)$. The effect of early granulosa cell removal on multiple PN incidence remains controversial. Ding et al. found that the normal fertilization rate when removing the granulosa cells after incubating the gametes for five hours was similar to conventional IVF, but the proportion of three PN embryos was significantly higher (10.9\% vs. 4.5\%) [29]. Other studies had similar findings [15][17]. However, He et al. found no difference in multiple PN rate [30], and Xiong et al. reported lower multiple PN rate (7.48\% vs. 9.22\%) [14] in IVF-S than conventional IVF. Because granulosa cells provide the oocyte with growth factors or adhesion molecules and play an important role in oocyte growth, maturation, and normal fertilization, early removal of granulosa cells might affect the second meiosis or cortical reaction during IVF. Another explaination for higher mutiple PN rate might be that a tiny minority of fertilized oocytes recognized as unfertilized, were performed R-ICSI. One of the most critical parts of the present study was to identify within six hours after conventional insemination those eggs that fail to fertilize. Therefore, one must rely on the correct identification of polar bodies. However, the eggs may be still under the process of fertilization despite the absence of 2PB after six hours of IVF incubation, which could lead to abnormal fertilization after R-ICSI. Payne et al showed that second

Page $10 / 15$ 
polar body was released in $\sim 90 \%$ of fertilized oocytes by $6 \mathrm{~h}$ and the maximum time course for $2 \mathrm{~PB}$ extrusion was $8 \mathrm{~h}$ which had very small percentage by time-lapse incubator [7]. Meanwhile, another study, which did 2PB evaluation after 6 $\mathrm{h}$ incubation, showed that oocytes which were identified with one polar body but were not reinseminated by ICSI (50 eggs) did not develop pronuclei and did not show any other signs of fertilization [9]. Therefore, using six hours for observation of 2PB is convinced, even though a tiny minority of fertilized oocytes might be recognized as unfertilized. In such situation, time-lapse incubators will be performed in the future study for identifying fertilization. One interesting finding in this study was that the multiple PN rate following IVF-S in Group 1 was $10.42 \%$, slightly lower than the 12.6 and $13.34 \%$ reported in other studies [15][17]. A possible explanation for this difference could be the fact that the early granulosa cell removal after short gamete coincubation in our study was performed in only four or five oocytes/zygotes per patient, rather than all oocytes/zygotes. If two or more oocyte/zygote was fertilized, the rest were cultured as conventional IVF. This reduced the proportion of early granulosa cells removal.

What is more, this study showed that the effectiveness of early R-ICSI after IVF-S was similar to conventional ICSI. As shown in Supplemental Table 1, early R-ICSI and the conventional ICSI in Group 2 were similar in the rates of normal fertilization, cleavage, d3 high-quality cleavage embryo and high-quality blastocyst. It was important to show that clinical pregnancy rate, implantation rate and live birth rate were comparable between early R-ICSI and regular ICSI [10]. Meanwhile, early R-ICSI was shown to be safe for the offspring. A study with large number cases was to investigate the effects of early cumulus cell removal $(n=570)$ on pregnancy and neonatal outcomes as compared to routine cumulus cell removal $(n=1214)$, and it showed that early cumulus cell removal alone was not associated with adverse pregnancy and neonatal outcomes [31]. Also, no differences in sex, birth weight, and birth defects were found between newborns delivered by conventional IVF, ICSI, or early R-ICSI [32][33]. However, possible risks associated with the extra manipulation during fertilization assessment after IVF-S still exist. Therefore, only four or five oocytes/zygotes per patient, rather than all oocytes/zygotes, were chosen for polar body observation. The method to reduce proportion of early granulosa cells removal, might help attenuated the risks.

Here, we suggest that early R-ICSI and split IVF-ICSI are effective methods to reduce IVF fertilization failure or low fertilization in couples with high-risk factors for poor fertilization. We believe that split IVF-ICSI is effective for high-risk patients in the first ART, but in this method more oocytes are fertilized by ICSI. From the economic [34] and offspring safety perspectives [35][36], early R-ICSI is preferred. Although this approach increased the multiple PN incidence, it was similar to split IVF-ICSI in rates of normal fertilization, normal cleavage, high-quality embryo, clinical pregnancy, and birth. The limitation of this study is a retrospective design. Here, we use propensity score matching to eliminate the influence of baseline differences in this real-world study. Another limitation of this study is the relatively low statistical power (25.87\%), which may be due to the small sample size. A much larger samples maybe including multiple centers are needed. Also, it would be better to do randomized controlled studies.

\section{Conclusions}

In conclusion, IVF-S with early R-ICSI and split IVF-ICSI are both effective strategies in preventing IVF fertilization failure or low fertilization rate in couples with high-risk factors. IVF-S with early R-ICSI could become the preferred approach because of its advantages-fewer ICSI procedures, similar clinical pregnancy rate and similar live birth rate.

\section{Abbreviations}

IVF-S: Short gamete coincubation in in vitro fertilization; R-ICSI: rescue intracytoplasmic sperm injection; ART: assisted reproductive technology; COCs: oocyte cumulus complexes; PCOS: polycystic ovary syndrome; GnRH : gonadotropinreleasing hormone; hCG: human chorionic gonadotropin; PSM: propensity score matching; PN: pronuclei

Page $11 / 15$ 


\section{Declarations}

\section{Ethics approval and consent to participate}

All procedures performed in this study followed the ethical standards of the Ethical Review Board (ERB) of Sun Yat-sen Memorial Hospital (No. SYSEC-KY-KS-2021-123). The data were anonymized, and the requirement for informed consent was therefore waived.

\section{Consent for publication}

Not applicable.

\section{Availability of supporting data}

The data sets supporting the results of this article are included within the article and its additional files.

\section{Competing interests}

All authors declared no conflict of interest.

Funding: Grant support was provided by: the National Natural Science Foundation of China (81901451); Science and Technology Projects of Guangzhou, China (201704020046); Special Funds for Clinical Medical Research of Chinese Medical Association-Research and Development of Young Physicians in Reproductive Medicine (18010170746); the 5010 Programs Fund for clinical medicine research at Sun Yat-sen University (2014005).

\section{Authors' contributions}

LL $\mathrm{J}$ was responsible for experiment conception, data collection and analysis. YF Q and $\mathrm{XL} C$ was responsible for data collection and management. XH J, SB $O$ and RQ $L$ were responsible for data collection and analysis. $Y L$ and $D Z Y$ designed the work, provided technical guidance and final approved of manuscript. All authors read and approved the final manuscript

Acknowledgments: The authors thank all the doctors, nurses, and embryologists in the Reproductive Medicine Center of Sun Yat-sen Memorial Hospital for their help in collecting data.

\section{References}

1. Ming L, Liu P, Qiao J, Lian Y, Zheng X, Ren X, et al. Synchronization between embryo development and endometrium is a contributing factor for rescue ICSI outcome. Reprod Biomed Online. 2012;24:527-31.

2. Combelles CM, Morozumi K, Yanagimachi R, Zhu L, Fox JH, Racowsky C. Diagnosing cellular defects in an unexplained case of total fertilization failure. Hum Reprod. 2010;25:1666-71.

3. Shalom-paz E, Alshalati J, Shehata F, Jimenez L, Son WY, Holzer H, et al. Clinical and economic analysis of rescue intracytoplasmic sperm injection cycles. Gynecol Endocrinol. 2011;27:993-6.

4. Kuczynski W, Dhont M, Grygoruk C, Pietrewicz P, Redzko S, Szamatowicz M. Rescue ICSI of unfertilized oocytes after IVF. Hum Reprod. 2002;17:2423-7.

5. Plachot M, Junca AM, Mandelbaum J, Cohen J, Salat-Baroux J, Da Lage C. Timing of in-vitro fertilization of cumulusfree and cumulus-enclosed human oocytes. Hum Reprod. 1986;1:237-42. 
6. Chen $\mathrm{C}$, Sathananthan $\mathrm{AH}$. Early penetration of human sperm through the vestments of human eggs in vitro. Arch Androl. 1986;16:183-97.

7. Payne D, Flaherty SP, Barry MF, Matthews CD. Preliminary observations on polar body extrusion and pronuclear formation in human oocytes using time-lapse video cinematography. Hum Reprod. 1997;12:532-41.

8. Liu J, Zhang X, Yang Y, Zhao J, Hao D, Zhang J, et al. Long-time vs. short-time insemination of sibling eggs. Exp Ther Med. 2016;12:3756-60.

9. Nagy ZP, Rienzi LF, Ubaldi FM, Greco E, Massey JB, Kort HI. Effect of reduced oocyte aging on the outcome of rescue intracytoplasmic sperm injection. Fertil Steril. 2006;85:901-6.

10. Chen C, Kattera S. Rescue ICSI of oocytes that failed to extrude the second polar body $6 \mathrm{~h}$ post-insemination in conventional IVF. Hum Reprod. 2003;18:2118-21.

11. Liu W, Liu J, Zhang X, Han W, Xiong S, Huang G. Short co-incubation of gametes combined with early rescue ICSI: an optimal strategy for complete fertilization failure after IVF. Hum Fertil (Camb). 2014;17:50-5.

12. Kattera $\mathrm{S}$, Chen $\mathrm{C}$. Short coincubation of gametes in in vitro fertilization improves implantation and pregnancy rates: a prospective, randomized, controlled study. Fertil Steril. 2003;80:1017-21.

13. Le Bras A, Hesters L, Gallot V, Tallet C, Tachdjian G, Frydman N. Shortening gametes co-incubation time improves live birth rate for couples with a history of fragmented embryos. Syst Biol Reprod Med. 2017;63:331-7.

14. Xiong S, Han W, Liu JX, Zhang XD, Liu WW, Liu H, et al. Effects of cumulus cells removal after 6 h co-incubation of gametes on the outcomes of human IVF. J Assist Reprod Genet. 2011;28:1205-11.

15. Xue Y, Tong X, Jiang L, Zhu H, Yang L, Zhang S. Effect of cumulus cell removal $4 \mathrm{~h}$ post-insemination on fertilization and embryo quality: a prospective randomized sibling-oocyte study. J Assist Reprod Genet. 2013;30:1049-53.

16. Jin HX, Xin ZM, Song WY, Dai SJ, Sun YP. Effects of human cumulus cells on in vitro fertilization outcomes and its significance in short-term insemination. J Reprod Med. 2013;58:51-4.

17. Zhou L, Wang J, Xiao L, Sun H, Wang Y, Geng L, et al. Differential effects of short co-incubation of gametes and early removal of cumulus cells in patients with different fertilizing capabilities. Reprod Biomed Online. 2016;32:591-6.

18. Xiang W, Cong F, Lei JIA. Impact of Early Removal of Cumulus Cells from Cumulus-oocyteComplexes after Short-term Insemination on Embryo Qualityand Pregnant Outcome. JOURNAL OF SUN YAT-SEN UNIVERSITY (MEDICAL SCIENCES). 2014;35:390.

19. Hershlag A, Paine T, Kvapil G, Feng H, Napolitano B. In vitro fertilization-intracytoplasmic sperm injection split: an insemination method to prevent fertilization failure. Fertil Steril. 2002;77:229-32.

20. Goswami G, Gouri MD. Relevance of Split In vitro Fertilization-Intracytoplasmic Sperm Injection Method of Insemination in Normozoospermic and Mildly Oligospermic Men: A Retrospective Study. J Hum Reprod Sci. 2020;13:145-9.

21. Practice Committees of the American Society for Reproductive M, the Society for Assisted Reproductive Technology. Electronic address asrm asrm org. Intracytoplasmic sperm injection (ICSI) for non-male factor indications: a committee opinion. Fertil Steril. 2020;114:239-45. 
22. Moawad A, Abou-Ria c H, Elzaher d MA, Mohamed a. Split ICSI/Insemination in Mild Male Factor Infertility: a Randomized Controlled Trial. Egyptian Journal of Hospital Medicine [Internet]. 2011; Available from: http://egyptianjournal.net78.net/43_2.pdf

23. Hwang JL, Seow KM, Lin YH, Hsieh BC, Huang LW, Chen HJ, et al. IVF versus ICSI in sibling oocytes from patients with polycystic ovarian syndrome: a randomized controlled trial. Hum Reprod. 2005;20:1261-5.

24. Sauerbrun-Cutler MT, Huber WJ, Has P, Shen C, Hackett R, Alvero R, et al. Is intracytoplasmic sperm (ICSI) better than traditional in vitro fertilization (IVF): confirmation of higher blastocyst rates per oocyte using a split insemination design. J Assist Reprod Genet. 2020;37:1661-7.

25. Heikinheimo O, Bitzer J, Garcia Rodriguez L. Real-world research and the role of observational data in the field of gynaecology - a practical review. Eur J Contracept Reprod Health Care. 2017;22:250-9.

26. Harris AL, Vanegas JC, Hariton E, Bortoletto P, Palmor M, Humphries LA, et al. Semen parameters on the day of oocyte retrieval predict low fertilization during conventional insemination IVF cycles. J Assist Reprod Genet. 2019;36:291-8.

27. Montag $\mathrm{M}$, Koster $\mathrm{M}$, van der Ven $\mathrm{K}$, Bohlen $\mathrm{U}$, van der Ven $\mathrm{H}$. The benefit of artificial oocyte activation is dependent on the fertilization rate in a previous treatment cycle. Reprod Biomed Online. 2012;24:521-6.

28. Alpha Scientists in Reproductive M, Embryology ESIG of. The Istanbul consensus workshop on embryo assessment: proceedings of an expert meeting. Hum Reprod. 2011;26:1270-83.

29. Chen-hui D, Jing W, Si-da WEI, Yan-wen XU, Can-quan Z. Effects of Early Cumulus Cells Removal on In Vitro Fertilization in Short-Term Insemination. JOURNAL OF SUN YAT-SEN UNIVERSITY (MEDICAL SCIENCES). 2016;37:71.

30. He Y, Liu H, Zheng H, Li L, Fu X, Liu J. Effect of early cumulus cells removal and early rescue ICSI on pregnancy outcomes in high-risk patients of fertilization failure. Gynecol Endocrinol. 2018;34:689-93.

31. Kong P, Yin M, Tang C, Zhu X, Bukulmez O, Chen M, et al. Effects of Early Cumulus Cell Removal on Treatment Outcomes in Patients Undergoing In Vitro Fertilization: A Retrospective Cohort Study. Front Endocrinol (Lausanne). 2021;12:669507.

32. Huang B, Qian K, Li Z, Yue J, Yang W, Zhu G, et al. Neonatal outcomes after early rescue intracytoplasmic sperm injection: an analysis of a 5-year period. Fertil Steril. 2015;103:1432-7 e1.

33. Xiong F, Sun Q, Li G, Yao Z, Chen P, Wan C, et al. Perinatal and neonatal outcomes of pregnancies after early rescue intracytoplasmic sperm injection in women with primary infertility compared with conventional intracytoplasmic sperm injection: a retrospective 6-year study. BMC Pregnancy Childbirth. 2020;20:460.

34. Vitek WS, Galarraga O, Klatsky PC, Robins JC, Carson SA, Blazar AS. Management of the first in vitro fertilization cycle for unexplained infertility: a cost-effectiveness analysis of split in vitro fertilization-intracytoplasmic sperm injection. Fertil Steril. 2013;100:1381-8.

35. Esteves SC, Roque M, Bedoschi G, Haahr T, Humaidan P. Intracytoplasmic sperm injection for male infertility and consequences for offspring. Nat Rev Urol. 2018;15:535-62.

36. Pereira N, O'Neill C, Lu V, Rosenwaks Z, Palermo GD. The safety of intracytoplasmic sperm injection and long-term outcomes. Reproduction. 2017;154:F61-70. 


\section{Supplementary Files}

This is a list of supplementary files associated with this preprint. Click to download.

- SupplementalTablel.docx 